\title{
PROBIÓTICO (ENTEROCOCCUS FAECIUM) ADICIONADO A DIETAS ESTÁNDAR Y CON BAJA PROTEÍNA PARA CERDOS
}

\author{
PROBIOTIC (ENTEROCOCCUS FAECIUM) ADDED TO STANDARD AND LOW-PROTEIN \\ DIETS FOR PIGS
}

\author{
Reyes, I. ${ }^{1}$, Figueroa, J.L. ${ }^{*}$, Cobos, M.A. ${ }^{1}$, Sánchez-Torres, M.T. ${ }^{1}$, Zamora, V. ${ }^{1}$ \\ y Cordero, J.L. ${ }^{1}$
} Programa de Ganadería. Campus Montecillo. Colegio de Postgraduados. Montecillo. Texcoco. Estado de
México. México. *jlfigueroa@colpos.mx

Palabras clave adicionales

Canal cerdo. Urea plasmática.

\section{RESUMEN}

Se evaluó el comportamiento productivo (ganancia diaria de peso, GDP; consumo de alimento, COA; conversión alimenticia, CA; ganancia de carne magra, GCM), las características de la canal (área del músculo Longissimus, AML; grasa dorsal, GD; porcentaje de carne magra, CM), la concentración de urea en plasma, y la población microbiana en heces de 36 cerdos machos castrados híbridos (Yorkshire $\times$ Duroc $\times$ Pietrain) en iniciación $(11,10 \pm 0,51 \mathrm{~kg})$, crecimiento $(25,75 \pm$ $1,13 \mathrm{~kg})$, y finalización $(51,03 \pm 2,20 \mathrm{~kg})$, en respuesta a dos niveles de proteína bruta (PB; iniciación: 20,5 y $16,0 \%$; crecimiento: 16,0 y 14,5 $\%$; y finalización: 14,0 y 12,5 \%) y dos niveles del probiótico (PRO) Enterococcus faecium ( 0,0 y 1,0 $\mathrm{kg} \mathrm{t}^{-1}$ de alimento en cada etapa) en dietas sorgopasta de soya, en un diseño completamente al azar con arreglo factorial $2 \times 2$, con 9 repeticiones por tratamiento. La energía se mantuvo constante en 3,265 Mcal kg-1 . En iniciación, la adición del PRO redujo $(p \leq 0,05)$ el AML, pero no afectó $(p>0,05)$ otras variables en estudio; la urea en plasma disminuyó $(p \leq 0,05)$ en cerdos alimentados con baja proteína. En crecimiento y finalización, al reducir la PB disminuyó $(p \leq 0,01)$ la urea en plasma, pero no afectó $(p>0,05)$ otras variables. Agregar PRO aumentó $(p \leq 0,05)$ la concentración de Enterococcus y el total de bacterias en heces en todas las etapas. Estos resultados indican que agregar PRO aumenta la población microbiana total y de Enterococcus, pero no afecta la respuesta productiva, las características de la canal,

\section{Additional KeYWORdS}

Pig carcass. Plasmatic urea.

ni la concentración de urea en plasma de cerdos alimentados con dietas estándar o con baja proteína.

\section{SUMMARY}

An experiment was conducted to evaluate the growth performance (average daily gain, ADG; average daily feed intake, ADFI; feed:gain ratio, FGR; fat free lean gain, FFLG), the carcass characteristics (Longissimus muscle area, LMA; backfat thickness, BFT; lean meat percentage, LMP), the plasma urea nitrogen concentration, and the fecal microbial population of 36 hybrid (Yorkshire $\times$ Duroc $\times$ Pietrain) barrows in nursery $(11.10 \pm 0.51 \mathrm{~kg})$, growing $(25.75 \pm 1.13 \mathrm{~kg})$, and finishing $(51.03 \pm 2.20 \mathrm{~kg})$ phases of fattening in response to two levels of crude protein (CP; nursery: 20.5 and $16.0 \%$; growing: 16.0 and 14.5 $\%$; and finishing: 14.0 and $12.5 \%$ ) and two levels of probiotic (PRO) Enterococcus faecium ( 0.0 and $1.0 \mathrm{~kg} \mathrm{t}^{-1}$ of food at each stage) in sorghumsoybean meal diets, in a completely randomized design with $2 \times 2$ factorial arrangement, with 9 replicates per treatment. The energy remained constant at $3.265 \mathrm{Mcal}_{\mathrm{kg}}{ }^{-1}$. In nursery pigs, the addition of $P R O$ reduced $(p \leq 0.05)$ LMA, but did not affect $(p>0.05)$ other variables. The plasma urea nitrogen decreased $(p \leq 0.05)$ in pigs fed lowprotein diets. In growing and finishing pigs, the reduction of CP decreased $(p \leq 0.01)$ plasma urea nitrogen concentration, but did not affect $(p>0.05)$ 
other variables. The addition of PRO increased $(p \leq 0.05)$ the concentration of Enterococcus and total fecal bacteria at all stages. These results indicate that adding PRO increases the total microbial population and the population of Enterococcus, although it does not affect the growth performan$\mathrm{ce}$, the carcass characteristics, and the concentration of plasma urea nitrogen of pigs fed standard or low-protein diets.

\section{INTRODUCCIÓN}

La contaminación ambiental ha provocado cambios en el clima mundial, por lo que uno de los temas prioritarios de investigación es buscar formas de disminuir este problema. La producción intensiva de cerdos contribuye con una parte de dicha contaminación ya que generan grandes cantidades de excretas y con ellas, de nutrientes no digeridos por el animal y desechos de su metabolismo. Por ello, se han buscado alternativas viables para evitar este problema. Una de estas alternativas es el uso de dietas con baja proteína bruta (DBP) adicionadas con aminoácidos (L-lisina.HCl, DL-metionina, L-treonina y L-triptófano), que permite disminuir la excreción de nitrógeno en heces y orina (Figueroa et al., 2004; Trujillo et al., 2007; Figueroa et al., 2008; Martínez et al., 2009). Esta alternativa tiene efectos positivos sobre el medio ambiente y el costo del alimento por unidad de ganancia de peso. Sin embargo, el aspecto negativo es la mayor deposición de tejido adiposo (Noblet et al., 1994) debido a la mayor cantidad de energía neta disponible (Le Bellego et al., 2001) que se observa con el aumento del grosor de la grasa dorsal; aunque la respuesta productiva es similar en cerdos en crecimiento alimentados con dietas estándar o con baja proteína (Figueroa et al., 2002), lo que se presume ocurre también en otras etapas de la engorda.

Los aditivos alimenticios permiten mejorar la respuesta animal mediante la modificación del metabolismo o del proceso digestivo, aumentando la eficiencia en la utilización de los nutrientes. Los probióticos son aditivos que mejoran el proceso digestivo al mantener un ambiente intestinal sano (Figueroa et al., 2006), ya que modifican la composición o la actividad de la microflora intestinal. Entre las bacterias probióticas importantes se encuentra Enterococcus faecium, que puede ser utilizada para prevenir problemas diarreicos en cerdos jóvenes, y que presumiblemente también puede tener un efecto benéfico en cerdos en crecimiento-finalización, al proporcionar un mejor ambiente intestinal que permite mayor aprovechamiento de los nutrientes.

Los objetivos de esta investigación fueron: evaluar el efecto de la adición del probiótico E. faecium en dietas estándar y con baja proteína sobre el comportamiento productivo, las características de la canal, la concentración de urea en plasma, y la concentración de bacterias totales y de Enterococcus en heces de cerdos en iniciación, crecimiento y finalización, alimentados con dietas a base de sorgo-pasta de soya.

\section{MATERIAL Y MÉTODOS}

Esta investigación se realizó en las instalaciones de la Unidad Porcina de la Granja Experimental del Colegio de Postgraduados, localizada en el municipio de Tecámac, Estado de México. Se utilizaron 36 cerdos machos castrados híbridos (Landracex Yorkshire $\times$ Duroc), con peso inicial de $11,10 \pm 0,51 \mathrm{~kg}$ en iniciación, $25,75 \pm 1,13 \mathrm{~kg}$ en crecimiento y $51,03 \pm 2,20 \mathrm{~kg}$ en finalización. Los animales se alojaron en corrales individuales de $1,2 \times 1,5 \mathrm{~m}$, con piso de concreto, equipados con comedero tipo tolva y bebedero de chupón, y se distribuyeron en un diseño completamente al azar con arreglo factorial $2 \times 2$, con dos niveles de proteína bruta (iniciación: 20,5 y 16,0\%; crecimiento: 16,0 y $14,5 \%$; y finalización: 14,0 y $12,5 \%$ ) y dos niveles del E. faecium $\left(0,0\right.$ y $1,0 \mathrm{~kg} \mathrm{t}^{-}$ ${ }^{1}$ de alimento) en cada etapa, con nueve repeticiones por tratamiento con un cerdo por unidad experimental. Los niveles de 


\section{USO DE PROBIÓTICOS EN CERDOS EN ENGORDA}

baja proteína se obtuvieron en investigaciones previas (iniciación: Trujillo et al., 2007; crecimiento: Martínez et al., 2009; finalización: Figueroa et al., 2008), y corresponden al nivel de proteína bruta en el cual la respuesta productiva es similar al de la dieta estándar. El alimento y el agua se ofrecieron ad libitum.

Las dietas (tabla I) se elaboraron con base a sorgo-pasta de soya, y se formularon con la misma concentración de energía metabolizable $\left(3,265 \mathrm{Mcal} \mathrm{kg}^{-1}\right)$. Los tratamientos (dietas) con baja proteína se adicionaron con AA sintéticos (L-lisina.HCL, DLmetionina, L-triptófano y L-treonina) hasta alcanzar los niveles recomendados por el NRC (1998) de la dieta estándar.

Al inicio y al final de cada etapa se controló el peso de los cerdos, y el consumo de alimento. Con esto se determinó la ganancia diaria de peso (GDP), el consumo diario de alimento (COA), y la conversión alimenticia (CA). También se midió con esa frecuencia la grasa dorsal (GD) y el área del músculo Longissimus (AML) a nivel de la décima costilla utilizando un ultrasonido de tiempo real Sonovet 600 con transductor abdominal de 3,5 MHz (Medison, Inc., Cypress, California, USA). Con esta información y con el peso inicial y final, se determinó la ganancia de carne magra (GCM) y el porcentaje de carne magra (PCM) en la canal con la ecuación del NPPC (1991). Para determinar la concentración de urea en plasma, se tomaron muestras de sangre en el día final de la etapa por medio de punción en la vena cava utilizando tubos vacutainer con heparina; las muestras se colocaron en hielo hasta que fueron centrifugadas a 2500 rpm (1286 g) durante 20 minutos para separar el plasma y las células sanguíneas. El plasma fue transferido a tubos de polipropileno y congelado a $-20^{\circ} \mathrm{C}$ hasta realizar las determinaciones de urea. También se obtuvieron muestras de heces al final de cada etapa, tomando aproximadamente $100 \mathrm{~g} \mathrm{di}$ rectamente del ano del cerdo mediante la técnica de estimulación anal (Gómez et al.,
2002). Las muestras se colocaron en hielo y posteriormente se conservaron a $-20^{\circ} \mathrm{C}$ hasta realizar la medición de la concentración de microorganismos viables y totales.

Las dietas experimentales se analizaron para determinar la energía bruta (EB) utilizando una bomba calorimétrica adiabática, y la proteína bruta (PB) mediante el método de Kjeldahl (AOAC, 1990); el calcio por medio de espectrofotometría de absorción atómica, y el fósforo por medio de espectrofotometría de absorción de rayos UV, siguiendo las metodologías de Fick et al. (1979). La determinación de la concentración de urea en plasma se realizó por espectrofotometría de absorción de rayos UV, de acuerdo a la metodología de Chaney y Marbach (1962).

La determinación de la concentración de E. faecium en heces se obtuvo a partir del número de unidades formadoras de colonias (UFC g-1) en cada muestra; la concentración total de bacterias en heces fue determinada por conteo directo (Cobos et al., 2007). Para realizar el análisis estadístico, los datos de la concentración de bacterias y el número de UFC fueron convertidos a logaritmo base 10 .

Los datos se analizaron de acuerdo al diseño mencionado. El análisis estadístico se realizó utilizando el procedimiento GLM de SAS (1998); además, se realizó la comparación de medias mediante la prueba de Tukey. También se calculó el precio de las dietas de cada tratamiento, con base al costo de los ingredientes en el tiempo de la realización del experimento.

\section{RESULTADOS}

\section{RespuestapRODUCTIVA, CARACTERÍSTICAS DELACANAL, Y CONCENTRACIÓNDEUREAEN PLASMA \\ Iniciación: No se observó efecto de la} adición del PRO (tabla II) o de la interacción $\mathrm{PB} \times \mathrm{PRO}$ sobre las variables productivas $(p>0,05)$. En cuanto a las características de la canal, la adición del PRO a la dieta dismi- 
REYES, FIGUEROA, COBOS, SÁNCHEZ-TORRES, ZAMORA Y CORDERO

Tabla I. Composición (\%) de las dietas utilizadas. (Composition (\%) of diets).

\begin{tabular}{|c|c|c|c|c|c|c|c|c|c|}
\hline \multirow{3}{*}{$\begin{array}{l}\text { Etapa } \\
\text { Tratamiento } \\
\text { Ingrediente }\end{array}$} & \multirow{2}{*}{\multicolumn{3}{|c|}{$\begin{array}{c}\text { Iniciación } \\
\text { Nivel de proteína NRC }\end{array}$}} & \multirow{2}{*}{\multicolumn{3}{|c|}{$\begin{array}{c}\text { Crecimiento } \\
\text { Nivel de proteína NRC }\end{array}$}} & \multirow{2}{*}{\multicolumn{3}{|c|}{$\begin{array}{c}\text { Finalización } \\
\text { Nivel de proteína NRC }\end{array}$}} \\
\hline & & & & & & & & & \\
\hline & Std & Baja & $(1998)^{a}$ & Std & Baja & $(1998)^{a}$ & Std & Baja & $(1998)^{a}$ \\
\hline Sorgo & 62,52 & 75,68 & - & 75,545 & 81,10 & - & 83,55 & 87,96 & - \\
\hline Pasta de soya (44\%) & 33,31 & 18,96 & - & 21,30 & 15,25 & - & 14,0 & 9,25 & - \\
\hline Aceite crudo de soya & 1,415 & 1,61 & - & 0,78 & 0,86 & - & 0,355 & 0,40 & - \\
\hline L-lisina $\mathrm{HCl}$ & 0,075 & 0,51 & - & 0,205 & 0,385 & - & 0,205 & 0,345 & - \\
\hline DL-metionina & 0,02 & 0,145 & - & 0,01 & 0,065 & - & - & 0,035 & - \\
\hline L-triptófano & - & 0,065 & - & - & 0,03 & - & - & 0,015 & - \\
\hline L-treonina & - & 0,18 & - & 0,015 & 0,09 & - & 0,01 & 0,07 & - \\
\hline Premezcla vitamínica ${ }^{b}$ & 0,125 & 0,125 & - & 0,125 & 0,125 & - & 0,125 & 0,125 & - \\
\hline Premezcla micromineralc ${ }^{c}$ & 0,125 & 0,125 & - & 0,125 & 0,125 & - & 0,125 & 0,125 & - \\
\hline Carbonato de calcio & 0,865 & 0,90 & - & 0,91 & 0,92 & - & 0,78 & 0,80 & - \\
\hline Ortofosfato & 1,10 & 1,25 & - & 0,735 & 0,80 & - & 0,60 & 0,625 & - \\
\hline Sal & 0,350 & 0,350 & - & 0,25 & 0,25 & - & 0,25 & 0,25 & - \\
\hline Probiótico (E. faecium) ${ }^{d}$ & - & - & - & - & - & - & - & - & - \\
\hline Antibiótico (oxitetrac.) & 0,10 & 0,10 & - & - & - & - & - & - & - \\
\hline TOTAL & 100,00 & 100,00 & - & 100,00 & 100,00 & - & 100,00 & 100,00 & - \\
\hline \multicolumn{10}{|l|}{ Análisis calculado (\%) } \\
\hline EM, Mcal kg-1 & 3,265 & 3,265 & 3,265 & 3,265 & 3,265 & 3,265 & 3,265 & 3,265 & 3,265 \\
\hline $\mathrm{PC}$ & 20,5 & 16,0 & 20,9 & 16,0 & 14,5 & 18,0 & 14,0 & 12,5 & 15,5 \\
\hline Calcio & 0,70 & 0,70 & 0,70 & 0,60 & 0,60 & 0,60 & 0,50 & 0,50 & 0,50 \\
\hline Fósforo total & 0,60 & 0,60 & 0,60 & 0,50 & 0,50 & 0,50 & 0,45 & 0,45 & 0,45 \\
\hline Fósforo disponible & 0,32 & 0,32 & 0,32 & 0,23 & 0,23 & 0,23 & 0,19 & 0,19 & 0,19 \\
\hline Lisina & 1,02 & 1,02 & 1,02 & 0,83 & 0,83 & 0,83 & 0,66 & 0,66 & 0,66 \\
\hline Treonina & 0,63 & 0,63 & 0,63 & 0,52 & 0,52 & 0,52 & 0,43 & 0,43 & 0,43 \\
\hline Triptófano & 0,18 & 0,18 & 0,18 & 0,15 & 0,15 & 0,15 & 0,12 & 0,12 & 0,12 \\
\hline Metionina + cistina & 0,58 & 0,58 & 0,58 & 0,47 & 0,47 & 0,47 & 0,39 & 0,39 & 0,39 \\
\hline Arginina & 0,42 & 0,42 & 0,42 & 0,33 & 0,33 & 0,33 & 0,24 & 0,24 & 0,24 \\
\hline Histidina & 0,32 & 0,32 & 0,32 & 0,26 & 0,26 & 0,26 & 0,21 & 0,21 & 0,21 \\
\hline Isoleucina & 0,55 & 0,55 & 0,55 & 0,45 & 0,45 & 0,45 & 0,37 & 0,37 & 0,37 \\
\hline Leucina & 1,02 & 1,02 & 1,02 & 0,83 & 0,83 & 0,83 & 0,67 & 0,67 & 0,67 \\
\hline Valina & 0,69 & 0,69 & 0,69 & 0,56 & 0,56 & 0,56 & 0,45 & 0,45 & 0,45 \\
\hline Fenilalanina + tirosina & 0,95 & 0,95 & 0,95 & 0,78 & 0,78 & 0,78 & 0,63 & 0,63 & 0,63 \\
\hline \multicolumn{10}{|l|}{ Análisis determinado (\%) } \\
\hline $\mathrm{PC}$ & 21,24 & 16,12 & - & 16,21 & 14,20 & - & 13,90 & 12,60 & - \\
\hline Calcio & 0,77 & 0,70 & - & 0,65 & 0,60 & - & 0,71 & 0,74 & - \\
\hline Fósforo total & 0,46 & 0,35 & - & 0,32 & 0,37 & - & 0,33 & 0,32 & - \\
\hline Costo dieta, USD kg-1e & 0,32 & 0,34 & - & 0,28 & 0,29 & - & 0,26 & 0,26 & - \\
\hline
\end{tabular}

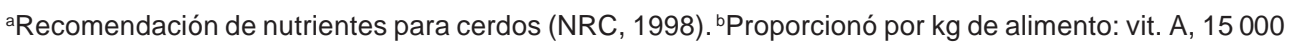
UI; vit. D3, $2500 \mathrm{UI}$; vit. E, 37,5 UI; vit. K, 2,5 mg; tiamina, 2,25 mg; riboflavina, 6,25 mg; niacina, $50 \mathrm{mg}$; piridoxina, 2,5 mg; cianocobalamina, 0,0375 mg; biotina, 0,13 mg; cloruro de colina, $563 \mathrm{mg}$; ácido pantoténico, $20 \mathrm{mg}$; ácido fólico, 1,25 mg. ${ }^{A}$ Aportó por kg de alimento: $\mathrm{Fe}, 150 \mathrm{mg} ; \mathrm{Zn}, 150 \mathrm{mg}$; Mn, 150 $\mathrm{mg} ; \mathrm{Cu}, 10 \mathrm{mg} ; \mathrm{Se}, 0,15 \mathrm{mg} ; \mathrm{I}, 0,9 \mathrm{mg} ; \mathrm{Cr}, 0,2 \mathrm{mg}$. dEl probiótico se agregó en lugar del antibiótico en dietas de iniciación; y disminuyendo en cantidad equivalente a la añadida, el sorgo en crecimiento y finalización. Las dietas de crecimiento y finalización no contenían antibiótico. ${ }^{e}$ Calculado en base a los precios de los ingredientes vigentes en octubre-diciembre de 2008. Convertido a dólares estadounidenses con la tasa vigente el 20 de enero del 2010.

Archivos de zootecnia vol. 61, núm. 236, p. 592. 


\section{USO DE PROBIÓTICOS EN CERDOS EN ENGORDA}

Tabla II. Respuesta productiva, características de la canal y concentración de urea en plasma de cerdos en iniciación alimentados con dietas estándar y con baja proteína adicionadas con probiótico. (Growth performance, carcass characteristics, and plasma urea nitrogen concentration of nursery pigs fed standard and low-protein diets supplemented with probiotic).

\begin{tabular}{|c|c|c|c|c|c|c|c|c|c|c|c|c|}
\hline $\mathrm{T}$ & PB & EM & GDP & $\mathrm{COA}$ & $C A$ & $\mathrm{PI}$ & PF & GCM & GD & AML & $\mathrm{CM}$ & U \\
\hline 1 & 20,5 & 3,265 & 0,56 & 1,07 & 1,90 & 11,08 & 26,28 & 0,21 & 0,31 & 11,03 & 45,64 & $22,91^{a}$ \\
\hline 2 & 20,5 & 3,265 & 0,50 & 0,95 & 1,94 & 11,15 & 24,33 & 0,17 & 0,28 & 9,28 & 45,16 & $26,81^{a}$ \\
\hline 3 & 16,0 & 3,265 & 0,57 & 1,11 & 1,93 & 11,12 & 26,41 & 0,21 & 0,34 & 10,92 & 45,22 & $13,61^{\mathrm{b}}$ \\
\hline 4 & 16,0 & 3,265 & 0,57 & 1,06 & 1,90 & 11,04 & 25,97 & 0,20 & 0,32 & 10,43 & 45,07 & $11,80^{\mathrm{b}}$ \\
\hline EEM & - & - & 0,02 & 0,04 & 0,09 & 0,10 & 0,82 & 0,01 & 0,01 & 0,45 & 0,47 & 1,98 \\
\hline \multicolumn{13}{|c|}{ Factores principales } \\
\hline \multirow[t]{2}{*}{ PB } & 20,5 & - & 0,53 & 1,01 & 1,92 & 11,12 & 25,31 & 0,19 & 0,30 & 10,15 & 45,40 & $24,86^{a}$ \\
\hline & 16,0 & - & 0,57 & 1,09 & 1,92 & 11,08 & 26,19 & 0,20 & 0,33 & 10,67 & 45,15 & $12,70^{b}$ \\
\hline \multirow[t]{2}{*}{ PRO } & 0,0 & - & 0,57 & 1,09 & 1,92 & 11,10 & 26,35 & 0,21 & 0,32 & $10,97^{a}$ & & 18,26 \\
\hline & 0,1 & - & 0,53 & 1,00 & 1,92 & 11,10 & 25,15 & 0,19 & 0,30 & $9,86^{b}$ & 45,12 & 19,30 \\
\hline \multicolumn{13}{|c|}{ Probabilidad del factor } \\
\hline PB & - & - & 0,25 & 0,19 & 0,95 & - & 0,44 & 0,27 & 0,10 & 0,35 & 0,64 & 0,01 \\
\hline PRO & - & - & 0,29 & 0,13 & 0,95 & - & 0,30 & 0,07 & 0,27 & 0,05 & 0,56 & 0,60 \\
\hline PB $\times$ PRO & - & - & 0,38 & 0,47 & 0,65 & - & 0,50 & 0,19 & 1,00 & 0,27 & 0,75 & 0,16 \\
\hline
\end{tabular}

ab Medias de tratamiento o efecto principal con distinta literal indica diferencias estadísticas $(p<0,05)$. $\mathrm{T}=$ Tratamiento; $\mathrm{PB}=$ Proteína bruta (\%); EM= Energía metabolizable $\left(\mathrm{Mcal} \mathrm{kg}^{-1}\right)$; GDP= Ganancia diaria de peso $\left(\mathrm{kg} \mathrm{d}^{-1}\right) ; \mathrm{COA}=$ Consumo de alimento $\left(\mathrm{kg} \mathrm{d}^{-1}\right) ; \mathrm{CA}=$ Conversión alimenticia; $\mathrm{PI}=\mathrm{Peso}$ inicial $(\mathrm{kg})$; $P F=$ Peso final $(\mathrm{kg}) ; \mathrm{GCM}=$ Ganancia de carne magra $\left(\mathrm{kg} \mathrm{d}^{-1}\right) ; \mathrm{GD}=$ Grasa dorsal $(\mathrm{cm}) ; A M L=$ Área del músculo Longissimus $\left(\mathrm{cm}^{2}\right) ; \mathrm{CM}=$ Carne magra $(\%) ; \mathrm{U}=$ urea en plasma $\left(\mathrm{mg} \mathrm{dL}^{-1}\right)$; $E E M=$ Error estándar de la media; $\mathrm{PRO}=$ Probiótico .

nuyó el AML $(\mathrm{p} \leq 0,05)$, pero se observó una tendencia $(\mathrm{p}=0,07)$ a incrementar la GCM en la canal. Por otro lado, el nivel de PB no afectó ( $\mathrm{p}>0,05$ ) el comportamiento productivo ni las características de la canal de los cerdos.

Al disminuir la PB de la dieta en 4,5 unidades porcentuales, la concentración de urea en plasma disminuyó $(\mathrm{p} \leq 0,01)$ en 48,91 \%; la adición del PRO a la dieta no afectó $(p>0,05)$ la concentración de urea plasmática.

Crecimiento. Las variables productivas no fueron afectadas $(\mathrm{p}>0,05)$ por el nivel del PRO o por la interacción del mismo con el nivel de PB (tabla III); sin embargo, se ob- servó una tendencia $(\mathrm{p}=0,07)$ a incrementar el COA por la interacción $\mathrm{PB} \times \mathrm{PRO}$.

En las características de la canal, sólo se observó una tendencia $(\mathrm{p}=0,06)$ a aumentar el AML al disminuir la PB en la dieta, y una tendencia $(\mathrm{p}=0,06)$ a disminuir cuando se agregó el PRO, pero no se encontraron efectos $(p>0,05)$ de la interacción PB $\times$ PRO. La concentración de urea en plasma se redujo $(\mathrm{p} \leq 0,01)$ 18,96\% al disminuir la PB en la dieta de 16 a 14,5\%. El nivel del PRO en la dieta no afectó $(p>0,05)$ la concentración de urea en plasma.

Finalización. En esta etapa (tabla IV) ninguna de las variables productivas o de la canal fueron afectadas $(\mathrm{p}>0,05)$ por el nivel 
REYES, FIGUEROA, COBOS, SÁNCHEZ-TORRES, ZAMORA Y CORDERO

Tabla III. Respuesta productiva, características de la canal y concentración de urea en plasma de cerdos en crecimiento alimentados con dietas estándar y con baja proteína adicionadas con probiótico. (Growth performance, carcass characteristics, and plasma urea nitrogen concentration of growing pigs fed standard and low-protein diets supplemented with probiotic).

\begin{tabular}{|c|c|c|c|c|c|c|c|c|c|c|c|c|}
\hline $\mathrm{T}$ & PB & EM & GDP & $\mathrm{COA}$ & $\mathrm{CA}$ & $\mathrm{PI}$ & PF & GCM & GD & AML & $\mathrm{CM}$ & $U$ \\
\hline 1 & 16,0 & 3,265 & 0,73 & 1,98 & 2,70 & 26,28 & 52,07 & 0,24 & 0,77 & 18,51 & 39,59 & 16,20 \\
\hline 2 & 16,0 & 3,265 & 0,66 & 1,73 & 2,60 & 24,33 & 47,77 & 0,22 & 0,67 & 16,09 & 39,62 & 15,45 \\
\hline 3 & 14,5 & 3,265 & 0,72 & 1,93 & 2,68 & 26,41 & 51,82 & 0,24 & 0,80 & 19,05 & 39,91 & 11,60 \\
\hline 4 & 14,5 & 3,265 & 0,75 & 2,00 & 2,67 & 25,97 & 52,46 & 0,25 & 0,80 & 18,49 & 39,43 & 14,05 \\
\hline EEM & - & - & 0,04 & 0,08 & 0,08 & 0,82 & 2,18 & 0,01 & 0,05 & 0,69 & 0,49 & 1,15 \\
\hline \multicolumn{13}{|c|}{ Factores principales } \\
\hline \multirow[t]{2}{*}{ PB } & 16,0 & - & 0,70 & 1,85 & 2,65 & 25,31 & 49,92 & 0,23 & 0,72 & 17,30 & 39,61 & $15,82^{\mathrm{a}}$ \\
\hline & 14,5 & - & 0,74 & 1,97 & 2,67 & 26,19 & 52,14 & 0,25 & 0,80 & 18,77 & 39,67 & $12,82^{b}$ \\
\hline \multirow[t]{2}{*}{ PRO } & 0,0 & - & 0,73 & 1,96 & 2,69 & 26,35 & $51, \varsigma$ & 0,2 & 0,78 & 18, & 39,75 & 13,90 \\
\hline & 0,1 & - & 0,71 & 1,86 & 2,63 & 25,15 & 50,12 & 0,24 & 0,73 & 17,29 & 39,53 & 14,75 \\
\hline \multicolumn{13}{|c|}{ Probabilidad del factor } \\
\hline PB & - & - & 0,31 & 0,20 & 0,78 & - & 0,32 & 0,16 & 0,14 & 0,06 & 0,89 & 0,01 \\
\hline PRO & - & - & 0,63 & 0,29 & 0,50 & - & 0,41 & 0,58 & 0,30 & 0,06 & 0,63 & 0,44 \\
\hline $\mathrm{PB} \times \mathrm{PRO}$ & - & - & 0,20 & 0,07 & 0,56 & - & 0,27 & 0,27 & 0,30 & 0,24 & 0,58 & 0,15 \\
\hline
\end{tabular}

abMedias de tratamiento o efecto principal con distinta literal indica diferencias estadísticas $(p<0,05)$. $\mathrm{T}=$ Tratamiento; $\mathrm{PB}=$ Proteína bruta (\%); EM= Energía metabolizable $\left(\mathrm{Mcal} \mathrm{kg}^{-1}\right) ; \mathrm{GDP}=$ Ganancia diaria de peso $\left(\mathrm{kg} \mathrm{d}^{-1}\right) ; \mathrm{COA}=$ Consumo de alimento $\left(\mathrm{kg} \mathrm{d}^{-1}\right) ; \mathrm{CA}=$ Conversión alimenticia; $\mathrm{PI}=\mathrm{Peso}$ inicial $(\mathrm{kg})$; $\mathrm{PF}=$ Peso final $(\mathrm{kg}) ; \mathrm{GCM}=$ Ganancia de carne magra $\left(\mathrm{kg} \mathrm{d}^{-1}\right) ; \mathrm{GD}=$ Grasa dorsal $(\mathrm{cm}) ; A M L=$ Área del músculo Longissimus $\left(\mathrm{cm}^{2}\right) ; \mathrm{CM}=$ Carne magra (\%); $\mathrm{U}=$ urea en plasma $\left(\mathrm{mg} \mathrm{dL}^{-1}\right)$; $\mathrm{EEM}=$ Error estándar de la media; $\mathrm{PRO}=$ Probiótico.

de PB o del PRO en la dieta, o por la interacción de ambos factores. Solamente la PB afectó $(\mathrm{p} \leq 0,01)$ la concentración de urea en plasma, observándose una reducción de $21,6 \%$ en la concentración de este metabolito al reducir la PB de la dieta de 14 a $12,5 \%$.

Concentración de bacterias en heces. La adición del probiótico (E. faecium) a las dietas, en todas las etapas del crecimiento de los cerdos, incrementó ( $\mathrm{p} \leq 0,05)$ la concentración de UFC y de bacterias totales en heces (tabla V). La proporción de bacterias del género Enterococcus representó menos del $4 \%$ del total de la población bacteriana presente en las heces en todas las etapas del crecimiento; incluso en la etapa de crecimiento, la población de bacterias de éste género fue menor al $1 \%$ (tabla VI).

\section{DISCUSIÓN}

\section{Respuesta productiva y CARACTERÍSTI- CAS DE LA CANAL Y UREA EN PLASMA}

La reducción de la proteína bruta en dietas para cerdos en iniciación, crecimiento y finalización, no afectó negativamente la respuesta productiva ni las características de la canal, y sólo disminuyó la concentración de urea en plasma, lo que coincide con reportes previos en los cuales utilizaron dietas sorgo-pasta de soya (Hansen et al., 


\section{USO DE PROBIÓTICOS EN CERDOS EN ENGORDA}

1993; Knowles et al., 1998; Figueroa et al., 2004; Trujillo et al., 2007); maíz-pasta de soya (Tuitoek et al., 1997; Mavromichalis et al., 1998; Figueroa et al., 2003); o trigomaíz-cebada (Le Bellego y Noblet, 2002). Otros autores han observado que alimentar cerdos con dietas con baja proteína aumenta la grasa corporal, lo que no ocurrió en este trabajo, probablemente porque dicha respuesta se observa en cerdos alimentados con maíz-pasta de soya (Kerr et al., 1995; Tuitoek et al., 1997).

La adición del probiótico a las dietas no mejoró la respuesta productiva, y sólo se encontró una tendencia a mejorar la GCM en iniciación (Díaz, 2006), y el COA en creci- miento (Chiquieri et al., 2006). En finalización, y en las demás variables de las otras etapas, no se encontró respuesta al probiótico, lo que no concuerda con lo reportado por Jeresiunas et al. (2006), quienes encontraron $6 \%$ más peso vivo en cerdos alimentados con este probiótico.

La reducción en la concentración de urea en plasma en todas las etapas de este experimento es muy notoria, aún con el nivel de proteína bruta utilizada, lo que coincide con otros autores (Ward y Southern, 1995; Knowles et al., 1998; Figueroa et al., 2002) e indica que no hay excesos de aminoácidos (Coma etal., 1995), por lo que hay una mayor eficiencia en la utilización de la proteína de

Tabla IV. Respuesta productiva, características de la canal y concentración de urea en plasma de cerdos en finalización alimentados con dietas estándar y con baja proteína adicionadas con probiótico. (Growth performance, carcass characteristics, and plasma urea nitrogen concentration of finishing pigs fed standard and low-protein diets supplemented with probiotic).

\begin{tabular}{|c|c|c|c|c|c|c|c|c|c|c|c|c|}
\hline $\mathrm{T}$ & PB & EM & GDP & $\mathrm{COA}$ & $C A$ & PI & PF & GCM & GD & AML & $\mathrm{CM}$ & $U$ \\
\hline 1 & 14,0 & 3,265 & 0,84 & 2,83 & 3,34 & 52,07 & 81,66 & 0,29 & 1,21 & 28,02 & 38,03 & 23,55 \\
\hline 2 & 14,0 & 3,265 & 0,85 & 2,50 & 2,93 & 47,77 & 81,61 & 0,30 & 1,21 & 27,52 & 37,84 & 21,74 \\
\hline 3 & 12,5 & 3,265 & 0,87 & 2,83 & 3,25 & 51,82 & 82,28 & 0,29 & 1,21 & 27,73 & 37,85 & 16,68 \\
\hline 4 & 12,5 & 3,265 & 0,84 & 2,73 & 3,30 & 52,46 & 82,00 & 0,29 & 1,22 & 27,05 & 37,60 & 19,07 \\
\hline EEM & - & - & 0,05 & 0,15 & 0,15 & 2,18 & 3,06 & 0,02 & 0,08 & 1,13 & 0,28 & 1,32 \\
\hline \multicolumn{13}{|c|}{ Factores principales } \\
\hline \multirow[t]{2}{*}{ PB } & 20,5 & - & 0,85 & 2,67 & 3,15 & 49,92 & 81,63 & 0,30 & 1,21 & 27,77 & 37,93 & $22,79^{a}$ \\
\hline & 16,0 & - & 0,85 & 2,78 & 3,28 & 52,14 & 82,14 & 0,29 & 1,21 & 27,39 & 37,72 & $17,87^{\mathrm{b}}$ \\
\hline \multirow[t]{2}{*}{ PRO } & 0,0 & - & 0,85 & 2,8 & 3,30 & 51,95 & $81, \varsigma$ & 0,29 & 1,2 & 27,88 & 37,94 & 20,11 \\
\hline & 0,1 & - & 0,84 & 2,62 & 3,13 & 50,12 & 81,79 & 0,29 & 1,21 & 27,88 & 37,72 & 20,55 \\
\hline \multicolumn{13}{|c|}{ Probabilidad del factor } \\
\hline PB & - & - & 0,91 & 0,47 & 0,34 & - & 0,87 & 0,77 & 0,96 & 0,75 & 0,47 & 0,01 \\
\hline PRO & - & - & 0,88 & 0,18 & 0,21 & - & 0,95 & 0,89 & 0,93 & 0,63 & 0,45 & 0,82 \\
\hline $\mathrm{PB} \times \mathrm{PRO}$ & - & - & 0,69 & 0,48 & 0,13 & - & 0,96 & 0,71 & 0,96 & 0,93 & 0,92 & 0,12 \\
\hline
\end{tabular}

abMedias de tratamiento o efecto principal con distinta literal indica diferencias estadísticas $(p<0,05)$. $\mathrm{T}=$ Tratamiento $\mathrm{PB}=$ Proteína bruta $(\%) ; \mathrm{EM}=$ Energía metabolizable $\left(\mathrm{Mcal} \mathrm{kg}^{-1}\right) ; \mathrm{GDP}=$ Ganancia diaria de peso $\left(\mathrm{kg} \mathrm{d}^{-1}\right) ; \mathrm{COA}=$ Consumo de alimento $\left(\mathrm{kg} \mathrm{d}^{-1}\right) ; \mathrm{CA}=$ Conversión alimenticia; $\mathrm{Pl}=\mathrm{Peso}$ inicial $(\mathrm{kg})$; $P F=$ Peso final $(\mathrm{kg}) ; \mathrm{GCM}=$ Ganancia de carne magra $\left(\mathrm{kg} \mathrm{d}^{-1}\right) ; \mathrm{GD}=$ Grasa dorsal $(\mathrm{cm}) ; A M L=$ Área del músculo Longissimus $\left(\mathrm{cm}^{2}\right) ; \mathrm{CM}=$ Carne magra (\%); $\mathrm{U}=$ urea en plasma ( $\mathrm{mg} \mathrm{dL}^{-1}$ ); $\mathrm{EEM}=\mathrm{Error}$ estándar de la media; $\mathrm{PRO}=$ Probiótico. 
Tabla V. Número de unidades formadoras de colonias y concentración de bacterias de muestras en heces de cerdos. (Amount of unities forming colonies and concentration of bacteria of faeces samples of pigs).

\begin{tabular}{cccc}
\hline $\mathrm{T}$ & UFC & $\begin{array}{c}\text { Bacterias } \\
\text { Enterococcus }\end{array}$ & $\begin{array}{c}\text { ( } \text { heces }) \\
\text { totales }\end{array}$ \\
\hline Iniciación & & & \\
1 & $19,00^{\mathrm{a}}$ & $9,500,000^{\mathrm{a}}$ & $1,59 \times 10^{11}$ \\
2 & $105,00^{\mathrm{b}}$ & $52,500,000^{\mathrm{b}}$ & $1,56 \times 10^{11}$ \\
3 & $33,00^{\mathrm{a}}$ & $16,875,000^{\mathrm{a}}$ & $1,34 \times 10^{11}$ \\
4 & $194,25^{\mathrm{b}}$ & $97,125,000^{\mathrm{b}}$ & $1,21 \times 10^{11}$ \\
Crecimiento & & & \\
1 & $4,00^{\mathrm{a}}$ & $2,000,000^{\mathrm{a}}$ & $2,09 \times 10^{11}$ \\
2 & $83,75^{\mathrm{b}}$ & $41,875,000^{\mathrm{b}}$ & $2,87 \times 10^{11}$ \\
3 & $6,75^{\mathrm{a}}$ & $3,375,000^{\mathrm{a}}$ & $2,43 \times 10^{11}$ \\
4 & $52,75^{\mathrm{b}}$ & $26,375,000^{\mathrm{b}}$ & $2,12 \times 10^{11}$ \\
Finalización & & & \\
1 & $29,00^{\mathrm{a}}$ & $14,500,000^{\mathrm{a}}$ & $1,96 \times 10^{11}$ \\
2 & $125,50^{\mathrm{b}}$ & $62,750,000^{\mathrm{b}}$ & $2,75 \times 10^{11}$ \\
3 & $12,00^{\mathrm{a}}$ & $6,000,000^{\mathrm{a}}$ & $1,96 \times 10^{11}$ \\
4 & $111,00^{\mathrm{b}}$ & $55,500,000^{\mathrm{b}}$ & $2,81 \times 10^{11}$ \\
\hline
\end{tabular}

${ }^{\mathrm{ab}}$ En cada columna dentro de cada etapa, medias con distinta literal indican diferencias estadísticas $(p<0,05)$.

$\mathrm{T}=$ tratamiento; $\mathrm{UFC}=$ Unidades formadoras de colonias, Enterococcus ( $\mathrm{g}^{-1}$ heces).

la dieta, adicionada con aminoácidos sintéticos (Zervas y Zijlstra, 2002). Lo anterior indica que hay una reducción en la excreción de nitrógeno en las excretas porcinas que puede ser de hasta $10 \%$ por cada unidad porcentual que se reduzca la proteína de la dieta (Ferket et al., 2002), lo que puede representar de 30-40\% menos nitrógeno en dietas con 3-4 \% menos proteína bruta (Bridges et al., 1995); y de 30 a $50 \%$ menos olor en explotaciones porcinas (Kerry Easter, 1995).

\section{ANÁLISIS MICROBIOLÓGICO}

La concentración de bacterias en heces varía de acuerdo a las características de la cepa utilizada y de factores externos de la granja. El lechón adquiere bacterias al naci- miento cuando inicia el amamantamiento (Vahjen et al., 2006) y de la microbiota fecal de la cerda, lo que lo hace funcional; pero la concentración de E. faecium se reduce una vez que el lechón ingiere alimento sólido (Macha et al., 2004). La adición del E. faecium a las dietas aumentó su concentración en heces, lo que coincide con reportes previos (Macha et al., 2004; Vahjen et al., 2006). El aumento en la concentración de esta bacteria se ha asociado con una disminución de diarreas después del destete (Simon, 2005), porque influye en la modulación de la respuesta inmune (Benavente, 2003; Zeyner y Boldt, 2006), lo que permite reducir o incluso prescindir de los antibióticos (Macedo, 2003).

Un beneficio adicional de la adición de probióticos a las dietas para cerdos es la disminución del nitrógeno amoniacal en heces (Chen et al., 2006), lo que refuerza el uso de dietas con baja proteína, ya que la combinación de ambas alternativas permite disminuir la excreción de nitrógeno de manera aditiva.

En conclusión, la adición del probiótico E. faecium a dietas sorgo-pasta de soya, estándar y con baja proteína, no afecta las variables productivas, las características de la canal, y la concentración de urea en plasma de cerdos en iniciación, crecimiento y finalización. La disminución de la proteína en la dieta no afectó negativamente la respuesta productiva ni las características de la canal; se tiene un efecto benéfico al redu-

Tabla VI. Concentración total de bacterias $y$ de bacterias viables del género Enterococcus. (Total concentration of bacteria and viable bacteria of Enterococcus).

\begin{tabular}{lccc}
\hline Etapa & $\mathrm{A}$ & $\mathrm{B}$ & $\mathrm{B}$ \\
& Total & Enterococcus & \\
\hline Inicio & $1,42 \times 10^{11}$ & $4,4 \times 10^{7}$ & 3,09 \\
Crecimiento & $2,38 \times 10^{11}$ & $1,8 \times 10^{7}$ & 0,76 \\
Finalización & $2,37 \times 10^{11}$ & $3,4 \times 10^{7}$ & 1,43 \\
\hline
\end{tabular}




\section{USO DE PROBIÓTICOS EN CERDOS EN ENGORDA}

cirse la concentración de urea en plasma en cerdos alimentados con dietas con baja proteína. El probiótico utilizado aumenta la población en heces de la bacteria adicionada en la dieta, así como la concentración total de la microflora.

\section{BIBLIOGRAFÍA}

AOAC. 1990. Official methods of analysis. $15^{\text {th }} \mathrm{ed}$. Association of Official Analytical Chemists. Arlington, VA. pp. 37-38.

Benavente, D.A. 2003. Efecto del probiótico Sprinter ${ }^{\circledR}$ en lechones recién nacidos. Tesis de Ingeniería en Ciencia y Producción Agropecuaria. Zamorano. Honduras. 22 pp.

Bridges, T.C., Turner, L.W., Cromwell, G.L. and Pierce, J.L. 1995. Modelling the effects of diet formulation on nitrogen and phosphorus excretion in swine waste. Appl Eng Agric, 11: 731-739.

Chaney, A.L. and Marbach, E.P. 1962. Modified reagents for determination of urea and ammonia. Clin Chem, 8: 130-132.

Chen, Y.J., Min, B.J., Cho, J.H., Kwon, O.S., Son, K.S., Kim, I.H. and Kim, S.J. 2006. Effects of dietary Enterococcus faecium SF68 on growth performance, nutrient digestibility, blood characteristics and faecal noxious gas content in finishing pigs. Asian-Aust. J Anim Sci, 19: 406-411.

Chiquieri, J.M.S., Soares, R.T.R.N., Souza, J.C.D., Hurtado Nery, V.L., Ferreira, R.A. y Ventura, B.G. 2006. Probiótico y prebiótico en la alimentación de cerdos en crecimiento y terminación. Arch Zootec, 55: 305-308.

Cobos, M.A., Pérez-Sato, M., Piloni-Martini, J., González, S.S. and Bárcena, J.R. 2007. Evaluation of diets containing shrimp shell waste and an inoculum of Streptococcus milleri on rumen bacteria and performance of lambs. Anim Feed Sci Technol, 132: 324-330.

Coma, J., Carrion, D. and Zimmerman, D.R. 1995. Use of plasma urea nitrogen as a rapid response criterion to determine the lysine requirement of pigs. J Anim Sci, 73: 472-481.

Díaz, D. 2006. El papel de Fecinor en la colonización y regeneración de la flora intestinal del lechón. Product Manager, Biotechnology Division Norel \& Nature. España. http://www.engormix.

\section{AGRADECIMIENTOS}

Esta investigación fue financiada por la Línea Prioritaria de Investigación $N^{\circ} 11$ del Colegio de Postgraduados, México. Los autores agradecen a NOREL de México S.A de C.V. por suministrar el probiótico.

com/s_articles_view.asp? $a r t=1030 \& A R E A=$ POR-165. (15/03/2009).

Ferket, P.R., Van Heugten, E., Van Kempen, T.A.T.G. and Angel, R. 2002. Nutritional strategies to reduce environmental emissions from nonruminants. J Anim Sci (E. Suppl. 2) 80: E168-E182.

Fick, K.A., McDowell, L.R., Miles, P.H., Wilkinson, N.S., Funk, J.D., Conrad, J.H. y Valdivia, R. 1979. Métodos de análisis de minerales para tejidos de plantas y animales. $2^{\mathrm{a}}$ edición. Departamento de Ciencia Animal. Universidad de Florida. Gainesville, Florida. EEUU. pp. 601-701.

Figueroa, J.L., Lewis, A.J., Miller, P.S., Fischer, R.L., Gómez, R.S. and Diedrichsen, R.M. 2002. Nitrogen metabolism and growth performance of gilts fed standard corn-soybean meal diets or low-crude protein, amino acid-supplemented diets. J Anim Sci, 80: 2911-2919.

Figueroa, J.L., Lewis, A.J., Miller, P.S., Fischer, R.L. and Diedrichsen, R.M. 2003. Growth, carcass traits, and plasma amino acid concentrations of gilts fed low-protein diets supplemented with amino acids including histidine, isoleucine, and valine. J Anim Sci, 81: 1529-1537.

Figueroa, J.L., Cervantes, M., Cuca, M. y Méndez, M. 2004. Respuesta de cerdos en crecimiento y finalización a dietas con baja proteína y energía. Agrociencia, 38: 383-394.

Figueroa, J.L., Chi, E., Cervantes, M. y Domínguez, I.A. 2006. Alimentos funcionales para cerdos al destete. Vet Méx, 37: 117-136.

Figueroa, J.L., Martínez, M., Trujillo, J.E., Zamora, V., Cordero, J.L. and Sánchez-Torres, M.T. 2008. Plasma urea nitrogen concentration and growth performance of finishing pigs fed sorghum-soybean meal, low-protein diets. $J$ Appl Anim Res, 33: 7-12.

Gómez, R.S., Lewis, A.J., Miller, P.S. and Chen, H.Y. 2002. Growth performance, diet apparent digestibility, and plasma metabolite of barrows 


\section{REYES, FIGUEROA, COBOS, SÁNCHEZ-TORRES, ZAMORA Y CORDERO}

fed corn-soybean meal diets or low-protein, amino acid supplemented diets at different feeding levels. J Anim Sci, 80: 644-653.

Hansen, J.A., Knabe, D.A. and Burgoon, K.G. 1993. Amino acid supplementation of lowprotein, sorghum-soybean meal diets for 20- to 50-kilogram swine. J Anim Sci, 71: 442-451.

Jeresiunas, A., Kulpys, J. and Rolandas, S. 2006. The influence of probiotic Enterococcus faecium on pigs fattening. Veterinarija ir Zootechnika T, 35: 53-57.

Kerr, B.J., and R.A. Easter. 1995. Effect of feeding reduced protein, amino acid-supplemented diets on nitrogen and energy balance in grower pigs. J Anim Sci, 73: 3000-3008.

Kerr, B.J., McKeith, F.K. and Easter, R.A. 1995. Effect on performance and carcass characteristics of nursery to finisher pigs fed reduced crude protein, amino acid-supplemented diets. J Anim Sci, 73: 433-440.

Knowles, T.A., Southern, L.L., Bidner, T.D., Kerr, B.J. and Friesen, K.G. 1998. Effect of dietary fiber or fat in low-crude protein, crystalline amino acid-supplemented diets for finishing pigs. J Anim Sci, 76: 2818-2832.

Le Bellego, L. and Noblet, J. 2002. Performance and utilization of dietary energy and amino acids in piglets fed low protein diets. Livest Prod Sci, 76: 45-58.

Le Bellego, L., Van Milgen, J., Dubois, S. and Noblet, J. 2001. Energy utilization of low-protein diets in growing pigs. J Anim Sci, 79: 12591271.

Macha, M., Taras, D., Vahjen, W., Arini, B. and Simon, O. 2004. Specific enumeration of the probiotic strain Enterococcus faecium NCIMB 10415 in the intestinal tract and in faeces of piglets and sows. Arc Anim Nutr, 58: 443-452.

Macedo, M.G. 2003. Probióticos: una fórmula para el futuro. Nutrición Clínica, 6: 419-433.

Martínez, M., Figueroa, J.L., Trujillo, J.E., Zamora, V., Cordero, J.L., Sánchez-Torres, M.T.y Reyna, L. 2009. Respuesta productiva y concentración de urea en plasma de cerdos en crecimiento alimentados con dietas sorgo-pasta de soya con baja proteína. Vet Mex, 40: 27-38.

Mavromichalis, I., Webel, D.M., Emmert, J.L., Moser,
R.L. and Baker, D.H. 1998. Limiting order of amino acids in a low-protein, corn-soybean meal-whey-based diet for nursery pigs. J Anim Sci, 76: 2833-2837.

Noblet, J., Fortune, H., Shi, X.S. and Dubois, S. 1994. Prediction of net energy value of feeds for growing pigs. J Anim Sci, 72: 344-354.

NPPC. 1991. Procedures to evaluate market hogs ( $3^{\text {rd }}$ ed.). National Pork Producers Council. Des Moines, IA. USA.

NRC. 1998. Nutrient Requirements of Swine. $10^{\text {th }}$ ed. National Academy Press. Washington, DC.

SAS. 1998. The SAS system for Windows. V. 8. SAS Institute Inc. Cary NC. US.

Simon, O. 2005. Micro-organisms as feed additives - Probiotics. Adv Pork Prod, 16: 161-167.

Trujillo, J.E., Figueroa, J.L., Martínez, M., Zamora, V., Cordero, J.L., Sánchez-Torres, M.T., Cuca, M. y Cervantes, M. 2007. Concentración de urea en plasma y respuesta productiva de cerdos en iniciación alimentados con dietas sorgo-pasta de soya bajas en proteína. Agrociencia, 41: 597-607.

Tuitoek, K., Young, L.G., De Lange, F.M. and Kerr, B.J. 1997. The effect of reducing excess dietary amino acids on growing-finishing pig performance: An evaluation of the ideal protein concept. J Anim Sci, 75: 1575-1583.

Vahjen, W., Taras, D. and Simon, O. 2006. Effect of the probiotic Enterococcus faecium NCIMB10415 on cell numbers of total Enterococcus spp., E. faecium and E. faecalis in the intestine of piglets. Curr Issues Intestinal Microbiol, 8: 1-8.

Ward, T.L. and Southern, L.L. 1995. Sorghum amino acid-supplemented diets for the 50- to 100-kilogram pig. J Anim Sci, 73: 1746-1753.

Zervas, S. and Zijlstra, R.T. 2002. Effects of dietary protein and oathull fiber on nitrogen excretion patterns and postprandial plasma urea profiles in grower pigs. J Anim Sci, 80: 3238-3246.

Zeyner, A. and Boldt, E. 2006. Effects of a probiotic Enterococcus faecium strain supplemented from birth to weaning on diarrhoea patterns and performance of piglets. J Anim Physiol Anim Nutr, 90: 25-31. 